\title{
Article
}

\section{Using GPS Trajectories to Adaptively Plan Bus Lanes}

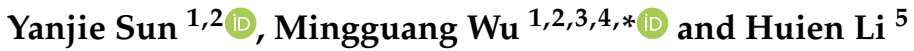 \\ 1 Key Laboratory of Virtual Geographic Environment of Ministry of Education, Nanjing Normal University, \\ Nanjing 210023, Jiangsu, China; 201301022@njnu.edu.cn \\ 2 College of Geographic Sciences, Nanjing Normal University, Nanjing 210023, Jiangsu, China \\ 3 State Key Laboratory Cultivation Base of Geographic Environment Evolution (Jiangsu Province), \\ Nanjing 210023, Jiangsu, China \\ 4 Jiangsu Center for Collaborative Innovation in Geographical Information Resource Development and Application, \\ Nanjing 210023, Jiangsu, China \\ 5 Jiangsu Institute of Geographic Information Industry, Nanjing 210023, Jiangsu, China; huienli1995@gmail.com \\ * Correspondence: wmg@njnu.edu.cn; Tel.: +86-25-8371-7160
}

check for updates

Citation: Sun, Y.; Wu, M.; Li, H. Using GPS Trajectories to Adaptively Plan Bus Lanes. Appl. Sci. 2021, 11, 1035. https://doi.org/10.3390/ app11031035

Academic Editor: Luís Picado Santos Received: 10 December 2020

Accepted: 20 January 2021

Published: 24 January 2021

Publisher's Note: MDPI stays neutral with regard to jurisdictional claims in published maps and institutional affiliations.

Copyright: (C) 2021 by the authors. Licensee MDPI, Basel, Switzerland. This article is an open access article distributed under the terms and conditions of the Creative Commons Attribution (CC BY) license (https:/ / creativecommons.org/licenses/by/ $4.0 /)$.

\begin{abstract}
Since bus prioritization policies can help mitigate urban traffic jams, the planning of bus lanes has drawn considerable attention. Existing methods suffer from a common limitation, which is that the limited spatial adaptability resulting from certain road condition information cannot be directly specified. Many bus GPS trajectories have been accumulated and can be contiguously gathered if needed. This paper proposes a trajectory-based bus lane planning method. First, we formulize the bus lane planning problem as a multiobjective optimization problem in which the road conditions, traffic flow, connectivity of bus lanes, and construction cost are organized as four constraints, and road utilization and bus punctuality are modeled as two objectives. Then, an evolutionary algorithm-based method is presented to solve the problem. We tested the model in the Nanshan District, Shenzhen City, China. Through a comparison with existing survey-based methods, the parameters associated with road conditions in this method are directly extracted from GPS trajectories, and this method is more effectively deployed than other methods. Since GPS trajectories can cover a wide area if needed, and because the proposed method can be effectively executed, this method can be adapted to large urban scales.
\end{abstract}

Keywords: traffic congestion; bus lane planning; GPS trajectory; multiobjective optimization

\section{Introduction}

Bus lanes play an important role in mitigating urban traffic congestion, which is a worldwide urban issue [1]. The purpose of building bus lanes is to give the right of way to bus systems and sustainably alleviate urban traffic congestion by attracting potential riders. As early as 1939, the first bus lane system in the world was constructed in Chicago [2]. Since then, due to their superiority in terms of the corresponding transportation capacity, construction cost, and construction period length, such systems have attracted widespread attention and research worldwide and have become widely accepted as a means of alleviating urban traffic congestion [3,4]. Under the guidance of bus prioritization, increasing numbers of cities have planned bus lanes [5,6]. For example, in China, more than two hundred cities, including Beijing, Shanghai, Shenzhen, and Nanjing, have constructed bus lanes. Determining how to effectively plan bus lanes has become a new concern, and planning methods that are adaptive to city scales are urgently needed in practice.

Considerable effort has been devoted to finding effective methods to plan bus lanes. The existing bus lane planning methods can be approximately divided into two groups: (1) micromodels that focus on the road segment scale or micronetwork links, and (2) macromodels that focus on the actual city scale. In the first group, Seo et al. [7] simulated the impact of the bus lane configuration on the traffic times of buses and cars based on a microscopic traffic simulation model, NETSIM (network simulation), which is a software 
package that can microscopically simulate vehicular movements in a street network. The results showed that the derived bus lanes are effective in easing traffic congestion in specific traffic flows. However, this simulation addressed two main arterial roads, and thus requires more experiments in a large city area. Mesbah et al. [8] implemented a bus lane planning model at the network level. The road capacity and initial travel time were specified according to experience, and the travel time and traffic flow were specified by a road impedance function (a common method used for traffic simulations regarding the traffic flow and travel time). Si et al. [9] built a multimodal road impedance function for bus lane planning. This approach can be used to obtain the traffic parameters in a multimodal network with complex traveler choice behaviors, including transportation and route choices. Generally, in micromodels, the parameters related to traffic information (e.g., travel time and flows) are obtained by experience or through traffic simulations. Except for a few models used in experiments involving a single urban road, most models have been tested only on an ideal transport network [10]. For example, Si, Ming, Yang, and Gao [9] tested a model with a simple four-node and five-link network, and Miandoabchi et al. [11] used a network with eight nodes and 13 links. Generally, there has been a lack of verification using complex road networks at the city scale. It is extremely computationally expensive to use traffic simulations to obtain detailed traffic parameters covering a large area [8]. Moreover, solving the model involves a complex combinatorial optimization problem that is generally nonlinear and NP-hard (Non-deterministic Polynomial), and the calculation time is unacceptable for large datasets. Thus, those methods cannot yet be adapted to large city scales.

In the second group, Bagloee and Ceder [12] built a transport network planning model for an actual road network. The model used a heuristic search to determine the optimal candidate road segments, and the method was tested in Winnipeg, Canada. Hadas and Nahum [13] designed a multiobjective programming model of bus lanes. The model considered various input parameters, including the traffic flow, travel time, and construction costs. A multiobjective evolutionary algorithm was used to find the optimal solutions, and the model was verified in an actual case in Israel. The above methods also use traffic simulation models to specify the needed traffic parameters, but they suffer from the common constraint of limited adaptability for practical use. In addition to simulation models, there have also been efforts to obtain traffic parameters, e.g., number of buses and bus routes, through onsite collection and questionnaires in the second group. For example, Khoo, Teoh, and Meng [10] designed a biobjective bus lane optimization model. Unlike those parameters in simulation-based models, the non-bus traffic flow and bus stop times in this model were specified on site. In practice, collecting traffic information on site requires effort and time. Indeed, these macromodels can be used for actual city-scale road networks. However, compared with micromodels, the macromodels lack consideration of the microscopic aspects of road segments, such as bus routes and the frequency and number of vehicles.

As discussed above, both types of methods suffer from a common limitation, namely, limited spatial adaptability, because the fundamental parameters of traffic conditions (e.g., vehicle speed and traffic flow) cannot be effectively specified. The existing micromodels have limited adaptability and cannot be directly applied to overall urban road networks due to the high complexity of the traffic simulation model. The existing macromodels lack consideration of the microscopic-scale characteristics of vehicles and bus lanes.

There have been many analysis applications with GPS trajectories, such as adaptive bus route planning $[14,15]$ and traffic pattern detection [16]. However, such a method has not yet been reported for bus lane planning. To fill this gap, this paper proposes an adaptive trajectory-based bus lane planning method for complex networks at the city scale. We observed that buses may now be equipped with a GPS, and a large number of bus GPS trajectories have been accumulated and can be contiguously gathered if needed. Bus GPS trajectories can be used to obtain road and vehicle information (e.g., vehicle speed and traffic flow) and then used to plan bus lanes. Accordingly, we formulate the bus lane 
planning problem as a multiobjective optimization problem. We feed the bus GPS trajectory information into this model and introduce a multiobjective optimization algorithm to solve the model. The remainder of this paper is organized as follows. Section 2 gives the multiobjective planning model for bus lane planning. Section 3 proposes a multiobjective evolutionary algorithm to solve the bus lane planning model. Section 4 discusses the choice of the Nanshan District, Shenzhen, as the research area to test the model. A discussion of the performance and adaptability of the model can be found in Section 5. Section 6 summarizes the main contributions and future work

\section{Problem Formulization}

This paper formulates the bus lane planning problem as a multiobjective optimization problem. Specifically, we consider four constraints, namely, the road conditions, construction budget, traffic flow, and number of bus lanes, and two objectives, namely, road utilization and bus punctuality. A detailed discussion follows.

\subsection{Constraints}

Upon comprehensively reviewing the present literature on transportation planning [12,17-21], four constraints are considered.

(1) Road conditions (C1). Building a bus lane partially occupies the road, and the original road needs to meet the transformation standard, which generally considers the number of lanes and road width [22]. Candidate roads are therefore filtered according to the road type through a process mathematically expressed as shown in Equation (1):

$$
\forall i \in E^{\prime}, t_{i} \in T
$$

where $E^{\prime}$ represents all the candidate road segments of the bus lane, $i$ is one candidate of road segments, $t_{i}$ is the road type for road segment $i$, and $T$ is the appropriate road type set.

(2) Traffic flow (C2). Generally, road segments with low traffic flows are not needed for reconstruction into bus lanes. In practice, some cities have also introduced corresponding traffic flow standards for bus lanes. For example, the bus flow limitation of bus lane planning is 90 vehicles/ $\mathrm{h}$ for three-lane roads and 120 vehicles/ $\mathrm{h}$ for two-lane roads in Shenzhen, China. A bus flow threshold is therefore established for each candidate road segment, and it is formulated as shown in Equation (2):

$$
\forall i \in E^{\prime}, f_{i} \geq F
$$

where $f_{i}$ is the bus flow of candidate road segment $i$ and $F$ is the bus flow threshold. In model implementation, with GPS trajectories matched to road segments, the traffic flow on each road segment can be directly specified.

(3) Bus lane continuity (C3). Truong et al. [23] and Xiaolin et al. [24] suggest that short-distance or discrete bus lanes can lead to the further deterioration of traffic conditions. Continuous bus lanes are also relatively advantageous in terms of construction, passenger comfort, and safety $[19,25]$. To accommodate continuous constraints on bus lanes, the numbers of continuous candidate road segments and continuous noncandidate road segments are restricted in our model. $E_{c}$ is a set of connected candidate road segments for bus lanes form continuous candidate road segments. $E_{c n o n}$ is a set of continuous noncandidate road segments with a minimum number of noncandidate road segments between two disconnected segments $E_{c}$. There is a limitation on the number of road segments for every $E_{c}$ and $E_{c n o n}$. These restrictions are denoted as shown in Equations (3) and (4):

$$
\begin{gathered}
E_{c} \geq k_{c} \\
E_{\text {cnon }} \geq k_{\text {cnon }}
\end{gathered}
$$

where $k_{c}$ and $k_{\text {cnon }}$ are the corresponding thresholds of numbers of continuous candidate road segments and continuous noncandidate road segments. 
(4) Construction budget (C4). The construction budget of bus lanes is often limited. In our model, for any road segment $i$, there is a construction cost $c_{i}$ for the bus lane. Correspondingly, there is a total construction budget $B$. In practice, because the cost $c_{i}$ of each road segment is generally correlated with the road length, the length constraint is therefore used to replace the construction budget constraints in our model. The problem is formulated as Equation (5):

$$
\sum_{i \in E^{\prime}} c_{i} \leq B
$$

\subsection{Objective Function}

In existing bus lane planning models, the objective functions are organized from two perspectives: space and time. From the spatial perspective, bus lanes should use road space effectively. From the temporal perspective, bus lanes should improve the travel times of passengers [26]. Correspondingly, two objective functions are considered in this model.

\subsubsection{Road Utilization}

We model road utilization as the degree of utilization of bus lanes by buses. The existing methods consider the utilization rate of bus lanes mainly from two aspects: the number of covered buses [10] and the bus frequency [8,27]. Additionally, taking into account the fact that bus routes and the number of buses on each road segment are different, continuous bus lanes help passengers have a comfortable experience. We therefore emphasize the impact of bus route continuity on the utilization of bus lanes. In total, three factors are considered in terms of road utilization: (1) number of buses covered by bus lanes, (2) bus frequency, and (3) continuity of bus routes. Then, we organize this information into an objective function based on the quantitative possibility of a road being a candidate road, which is called the score in this paper. First, to distinguish the scores of road segments with different numbers of buses, the scores of each road segment are calculated by summing the score of every trajectory covered by the road segment. Then, since bus trajectory does not directly record bus frequency information, we calculate every trajectory segment score for each bus passing through a road segment to reflect the bus frequency. Finally, we split the bus trajectories according to the covered continuous planned road segments and then calculate the score separately for each bus trajectory segment.

Inspired by the utilization rate function of bicycle lanes based on GPS trajectories from bicycles [19], the road utilization rate score of bus lanes is formulated based on the three factors in Equation (6):

$$
t r_{j} \cdot g=\sum_{s \in j} \alpha^{\frac{L_{s}}{L_{\min }}} \times \frac{L_{s}}{L_{\min }}
$$

where $t r_{j} \cdot g$ represents the road utilization score of trajectory $t r_{j}$ and $j$ is the set of continuous road segments that overlaps with trajectory $t r_{j}$ in the planned road segment set $E^{\prime} . s$ is one continuous road segment belonging to set $j . L_{S}$ is the length of the road segments $s$. The normalized length of the continuous segments is calculated as $L_{s} / L_{\min }$, where $L_{\min }$ is the minimum length of a road segment covered by the trajectories. By performing an exponential operation on the normalization time, when $\alpha>1.0$, the weight of the continuity of the trajectory will be high; when $\alpha<1.0$, the bus number and frequency will have relatively high weights; and when $\alpha=1.0$, the weights of the above three objectives, namely, the number of buses, the frequency and the continuity, are equals.

Finally, the maximum total road utilization score $E^{\prime} \cdot g$ is calculated for the total candidate planned road segment, determined by the superposition of each bus trajectory score. This score is formulated as Equation (7):

$$
\max E^{\prime} \cdot g=\sum_{t r_{j} \in T r \& t r_{j} \cap E^{\prime} \neq \varnothing} t r_{j} \cdot g
$$

where $\operatorname{Tr}$ is all the bus trajectories. 


\subsubsection{Bus Punctuality}

We model bus punctuality as the fluctuations in the bus travel time. Many studies have calculated bus punctuality rates based on GPS trajectories. For example, Barabino et al. [28] proposed a new user-oriented punctuality measure to evaluate bus performance, and Lin et al. [29] introduced the concept of the adjacent site punctuality rate (ASPR). The proposed bus punctuality model applied extreme value theory between adjacent sites based on a distribution function of the bus travel time. Because our model is based on road segments for bus lane planning, the bus punctuality rate on road segments based on the ASPR is defined in this article and is formulated as Equation (8).

$$
R_{i}=P\left\{\left|T_{i}-t_{i j}\right|>S_{i}\right\}
$$

where $R_{i}$ is the punctuality rate of road segment $i, t_{i j}$ is the $j$ th bus travel time on road segment $i, T_{i}$ is the average value of the travel time, and $S_{i}$ is the standard deviation. Assuming the prior distribution of ASPR is $\beta(a, b)$, and it is able to be inferred that the posterior distribution is $\pi(R \mid m) \sim \beta(m+a, n-m+b) \beta(a, b)$, with a given $1-\alpha$, the confidence interval of punctuality is $\left[R_{1}{ }^{*}, R_{2}{ }^{*}\right]$. The shortest interval is calculated, and its mean value is used as the punctuality value. More details are discussed in [29].

Based on the above bus punctuality rate on a road segment, the objective function of the bus punctuality rate can be formulated as Equation (9).

$$
\max \sum_{i \in E^{\prime}} R_{i}
$$

\subsection{Multiobjective Bus Lane Planning Model}

From the above discussion on the relevant constraints and objectives, we present a multiobjective bus lane planning model. For a given bus trajectory set $T r$, road network $G=(V, E)$, and total construction budget $B$, finding the desired bus lane requires the identification of a set of edges $E^{\prime} \in E$ that have the maximum road utilization score and bus punctuality rate score. Mathematically, this problem can be expressed as a multiobjective planning model, as shown in Equation (10).

$$
\left\{\begin{array}{ccc} 
& \max \sum_{\operatorname{tr}_{j} \cap E^{\prime} \neq \varnothing} \sum_{s \in t r_{j}} \alpha^{\frac{L_{s}}{L_{\text {min }}}} \times \frac{L_{s}}{L_{\text {min }}} & \left(Z_{1}\right) \\
\text { Z: } & \max \sum_{i \in E^{\prime}} R_{i}, R_{i}=P\left\{\left|T_{i}-t_{i j}\right|>S_{i}\right\} & \left(Z_{2}\right) \\
\forall i \in E^{\prime}, t_{i} \in T & \left(C_{1}\right) \\
\forall i \in E^{\prime}, f_{i} \geq F & \left(C_{2}\right) \\
E_{c} \geq k_{c} & \left(C_{3}\right) \\
& E_{\text {cnon }} \geq k_{\text {cnon }} & \left(C_{4}\right) \\
\sum_{i \in E^{\prime}} c_{i} \leq B & \left(C_{5}\right)
\end{array}\right.
$$

Here, the data on road and vehicle parameters required by the proposed model, such as the traffic flow, number of buses in the lane, bus frequency, and road travel time, can be obtained via the GPS trajectory. By this way, it is even possible to specify traffic parameters with high spatiotemporal resolutions. For example, the GPS trajectories can be used to directly and efficiently calculate the traffic flows at the lane scale [14,30]. Moreover, compared with the traditional method of acquiring traffic parameters by historical experience, monitoring points, or numerical simulations, the use of GPS data can effectively cover all the road networks in a city $[30,31]$

\section{Problem Solving}

\subsection{Multiobjective Optimization}

The existing methods of solving the above multiobjective model can be approximately divided into two groups: (1) brute force searching and (2) heuristic searching. Brute force 
searching can identify the optimal solution, but it is usually time-consuming. The time complexity of finding the optimal solution for the NP-hard problem may be exponential, which makes it difficult to solve models at the city scale. Heuristic searching has been widely used to solve various complex problems. This approach can effectively achieve satisfactory results. Many search algorithms have been proposed in this group, such as simulated annealing, tabu searching, genetic algorithms, and ant colony optimization [32]. Among them, evolutionary algorithms can efficiently find multiple Pareto-optimal solutions simultaneously in a single simulation run [33]. There have been many studies and applications that have used evolutionary algorithms in practical multiobjective decision-making problems.

With high-efficiency and uniform-distribution solutions, the nondominated sorting genetic algorithm-II (NSGA2) is one of the most widely-used multiobjective evolutionary algorithms [34], and it has been applied in various transportation optimization problems, such as vehicle scheduling [35]. As a multiobjective genetic algorithm (GA), except for the basic steps of GA, such as population coding, the design of the fitness function and genetic operations, fast nondominated sorting, and the crowding distance are the most notable features of the NSGA2 algorithm. In follow-up studies, NSGA2 is also extended to solve constrained many-objective optimization problems. This approach has been proved to be especially suitable for low-dimensional optimization problems with a complex distribution of Pareto fronts (a set of multiobjective optimization solutions that do not dominate each other [36]). Therefore, based on NSGA2, an algorithm adapted to solve the above multiobjective bus lane planning model is proposed.

\subsection{Solution Algorithm}

We proposed a three-stage algorithm to solve the problem. The stages included (1) initialization, i.e., coding road segments based on the genetic population and creating initial populations; (2) iteration, i.e., population iteration in the GA, including selection, crossover, and mutation; and (3) result output, i.e., output of the model results. These stages are explained as follows, and Algorithm 1 gives the pseudo-code of the solution algorithm.

Initialization. First, it is the road segments coding of GA. In GA, there are three main types of individual coding: (1) binary encoding, (2) permutation encoding, and (3) value encoding. To map road segments to individual codes, binary encoding is selected, where 1 means that the road segment is selected as a bus lane candidate solution, and 0 means that it is not selected. In this sense, the initial solutions with size $N$ are a set of binary characters corresponding to the road segment as the population $P_{0}$. Figure 1 shows an example of candidate road segments and the corresponding GA encoding. After generating the initial solution, it should verity the feasibility of the solutions. Here we followed the constraint-domination method proposed in [37]. It calculates the constraint violation value $C V(x)$ of each solution $x$ using the following Equation (11):

$$
C V(x)=\sum_{j}\left\langle\bar{g}_{j}(x)\right\rangle+\sum_{k}\left|\bar{h}_{k}(x)\right|
$$

where $g_{j}(x)$, which is inequality constraint, includes $C_{2}, C_{3}, C_{4}$, and $C_{5}$ constraints in the model, and $h_{k}(x)$, which is equality constraint, includes $C_{1}$ constraint in the model. $\bar{g}_{j}(x)$ and $\bar{h}_{k}(x)$ are the normalized constraint functions. For $\langle\alpha\rangle$, if $\alpha\langle 0,\langle\alpha\rangle=0$, else $\langle\alpha\rangle=|\alpha|$. The constraint violation value could be used in subsequent operations of elitist selection and creation of offspring population.

Then, we calculate the multiobjective score of $P_{0}$ and use the constraint violation value and multiobjective scores to obtain the individual nondominated sorting and crowding distance results. The details about nondominated sorting and crowding distance are discussed in Deb, Pratap, Agarwal, and Meyarivan [34].

Iteration. In NSGA2, selection, crossover, and mutation are basic operators for finding optimum solution. First it is the elitist selection operator's role to choose two individuals from parent populations. It is based on the constraint violation value, nondominated sorting, and crowding distance. Then, it is the crossover operator which is used to exchange 
parts of the encodings of two parent individuals. Crossover operators include single-point, two-point, and uniform crossover functions. In this paper, two-point crossover is selected. The mutation operator enhances individual diversity by changing individual codings with a small probability. Here, the binary mutation operator determines whether the road segment should be selected as a bus lane. Then, it calculates the constraint violation value and multiobjective scores of the two offspring individuals. Finally, repeating the above operators occurs until generating the offspring population $Q_{t}$ with size $N$.
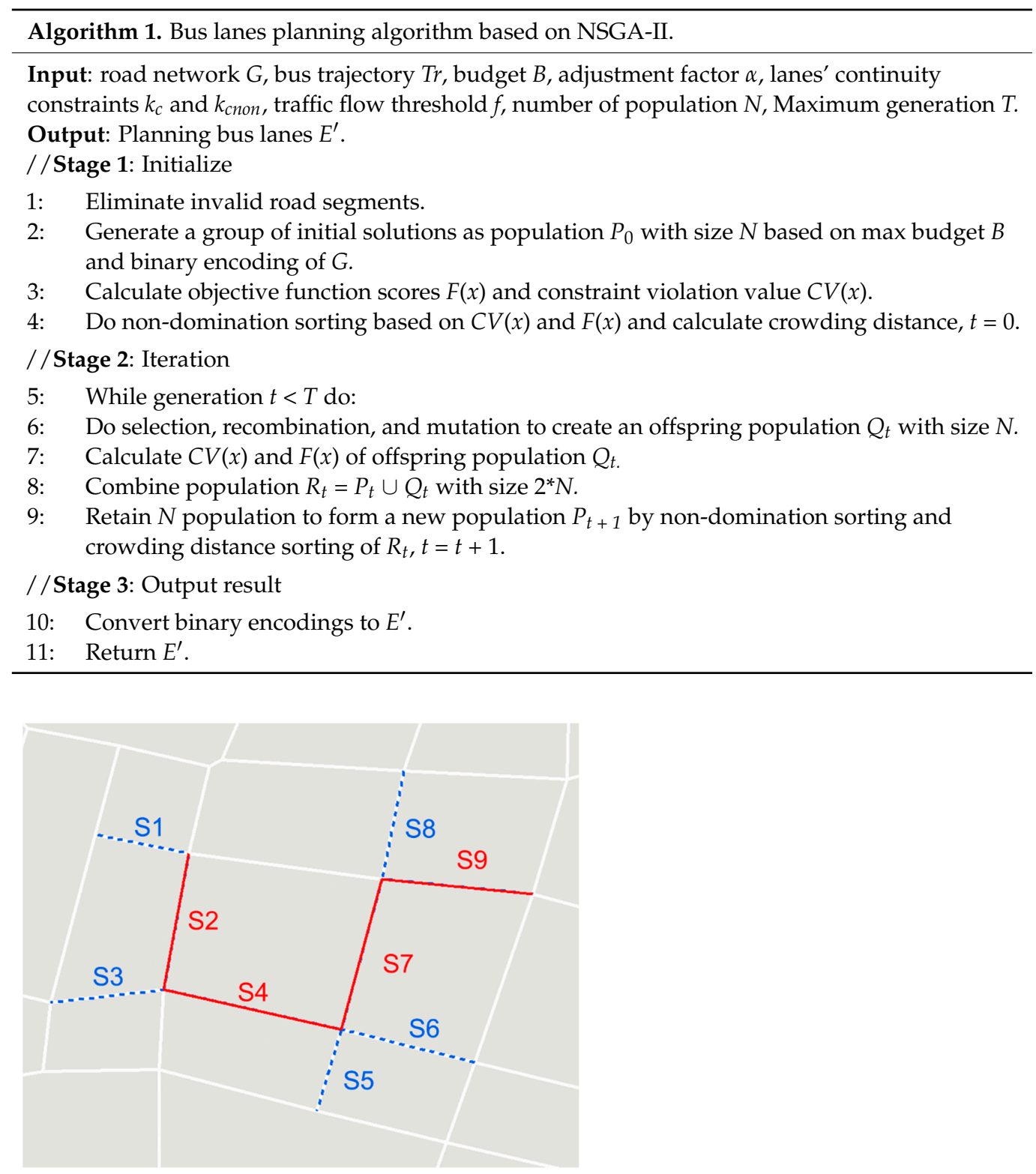

(a) Candidate road segments example

\begin{tabular}{|c|c|c|c|c|c|c|c|c|c|}
\hline Road ID & S1 & S2 & S3 & S4 & S5 & S6 & S7 & S8 & S9 \\
\hline GA code & 0 & 1 & 0 & 1 & 0 & 0 & 1 & 0 & 1 \\
\hline
\end{tabular}

(b) GA encoding example

Figure 1. Candidate road segments and genetic algorithm (GA) encoding example.

After one iteration, unlike traditional GA, the offspring population does not directly replace the parent population. First, the offspring and parent populations are merged into a group $R_{t}$ with size $2^{*} N$. Then, nondominated sorting and crowding distance values of all individuals are calculated based on constraint violation value and multiobjective 
scores. The new population is created by finding individuals with higher sorting ranks and crowding distances than the individuals in the previous population.

Output. If the generation is greater than the number of iterations, we terminate the iteration and output the result as the bus lane planning proposal.

\section{Experiments}

\subsection{Data Collection}

We selected Nanshan District, Shenzhen City, as the research area to test the model. The road network data were downloaded from OpenStreetMap, which is a website that provides free geographic data worldwide. Here, we used urban road network data and city point-of-interest (POI) data, including commercial areas, residential areas, and bus stations. Figure 2 shows the research area and the road network.

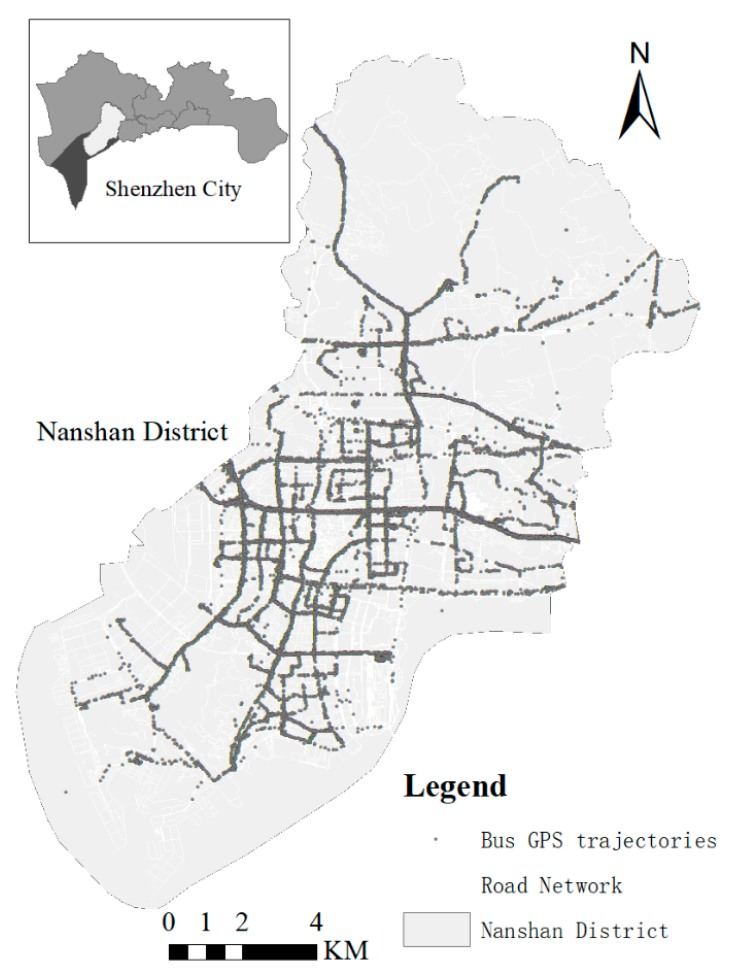

Figure 2. Research area overview.

The GPS trajectory data of buses were derived from the traffic management department of Shenzhen and were collected in March 2014. These data mainly include vehicle plate numbers, routes, and locations, as well as the acquisition times of GPS collection. Table 1 shows six example records.

Because bus lanes are mostly tidal lanes (bus lanes that are activated only during peak hours) [38], the bus trajectory data are selected from morning and evening peak times for two time periods (with the morning peak selected from 7:00 to 9:00, and the evening peak from 17:00 to 19:00), and the data spanned 7110 roads with 1952 bus trajectories.

Table 1. Examples of bus GPS data.

\begin{tabular}{ccccc}
\hline Plate Number & Routes & Longitude & Latitude & Acquisition Time \\
\hline BL8213 & 0E182 & 114.0091 & 22.58673 & $2014 / 3 / 67: 00: 06$ \\
BN3221 & M3433 & 113.9472 & 22.5839 & $2014 / 3 / 67: 00: 01$ \\
B90816 & M3583 & 113.969 & 22.558 & $2014 / 3 / 67: 00: 05$ \\
BM7988 & 03620 & 113.9453 & 22.52448 & $2014 / 3 / 67: 00: 02$ \\
BF7292 & B6824 & 113.905 & 22.53237 & $2014 / 3 / 67: 00: 02$ \\
BK4648 & B7364 & 113.9529 & 22.59186 & $2014 / 3 / 67: 00: 01$ \\
\hline
\end{tabular}




\subsection{Model Implementation}

\subsubsection{Preprocessing}

Considering the GPS data quality, we preprocessed the data before inputting the data into the proposed model, and this process mainly included three steps, as follows. (1) The trajectory data were analyzed, and discrete, large-error GPS points were removed [39]. (2) Multiple lanes were merged. Most road segments are related to multiple lanes. It is useful to know exactly how many lanes are on each road segment. However, these road attributes are not directly recorded in OpenStreetMap data. Therefore, a road segment with multiple lanes was merged into a single-lane road. (3) GPS trajectory points were matched to roads. Here, the interactive voting-based map matching algorithm (IVVM) [40] was selected for data matching. The algorithm, which considers the context information of the GPS sampling points, the mutual influence between the sampling points, and the attenuation of the influence with distance, has high precision for low-frequency GPS sampling points.

\subsubsection{Parameter Settings}

The input parameters of the model were set as follows: maximum construction budget $B=20 \mathrm{~km}$, continuity constraint $k_{c}=6, k_{\text {cnon }}=2$, adjustment factor $\alpha=1.0$, and traffic flow limit $f=90$ vehicles $/ \mathrm{h}$, as used in reality for bus lane planning in Shenzhen. To execute the GA, the maximum generation number $T$ was 1000 , population number $N$ was 200, crossover rate was 0.5 , and mutation rate was 0.1 . A high mutation rate may hinder the convergence of the genetic process to the true optimal Pareto front. The effects of these parameters on the results are discussed in Section 5.2. In addition, the algorithms were developed in the $\mathrm{C}++$ language, and a PC with $2.80 \mathrm{GHz}$ and $16.00 \mathrm{~GB}$ of RAM was used for the experiment.

\subsection{Results and Evaluation}

The Nanshan District road network and bus trajectory points matched to the corresponding road segments were used as input data. The algorithm requires $10 \mathrm{~min}$ to complete 1000 iterations. Figure 3 depicts the evolution of multiobjective scores in 1000 iterations. The two objective function scores rapidly increase at early iterations. After approximately 500 iterations, both objective function score curves exhibit a plateau, indicating that the results have converged.

Each point on the Pareto front in Figure 4 represents a nondominated bus lane proposal. Figure 5 shows three planning schemes with different objective preferences. Plan A tended to have the best bus punctuality rate. Additionally, Figure 4 illustrates that the road utilization rate of Plan A is the worst. Plan C tended to optimize the utilization of bus lanes the most, and the corresponding punctuality rate score was the lowest. Plan B is a balance between punctuality and utilization.

The following points can be observed from Figure 5. (1) In the best case of the punctuality rate, there are many small branches in the planning result of Plan A. In the multiobjective balanced and optimal utilization cases, there are several long road segment sets in the planned bus lane scheme because the objective function of bus utilization incorporates the continuity of bus routes. (2) Overlaying the results with subway lines and sites reveals that the model results are highly connected to the subway, which is consistent with the complementarity of subways and buses in urban transportation systems.

To illustrate the usability of the planning results, the results from the model were compared with the results of actual bus lane planning in Shenzhen, which is driven by historical experience and questionnaires organized by the bus management department. The balanced planning scheme created based on the proposed method was selected for comparison with the actual bus lane planning scheme that is implemented by the Transportation Bureau of Shenzhen Municipality. Figure 6 shows that the balanced planning scheme is largely consistent with the actual planning scheme. However, there are three road segments that are different from the case in the actual planning scheme. We used street-view images (which were collected in 2017 after the actual planning scheme was 
implemented) to investigate these differences. It can be observed from the street view that road segment (a) and road segment (c) have been planned as bus lanes, and road segment (b) is a two-lane road. In the proposed model, only roads with more than two lanes can be candidates. In practice, as in the test area, there is also a high demand for bus lanes on some two-lane roads, even though the planning of bus lanes on two-lane roads uses more traffic resources. Thus, the lane number threshold for bus lane planning needs further examination.

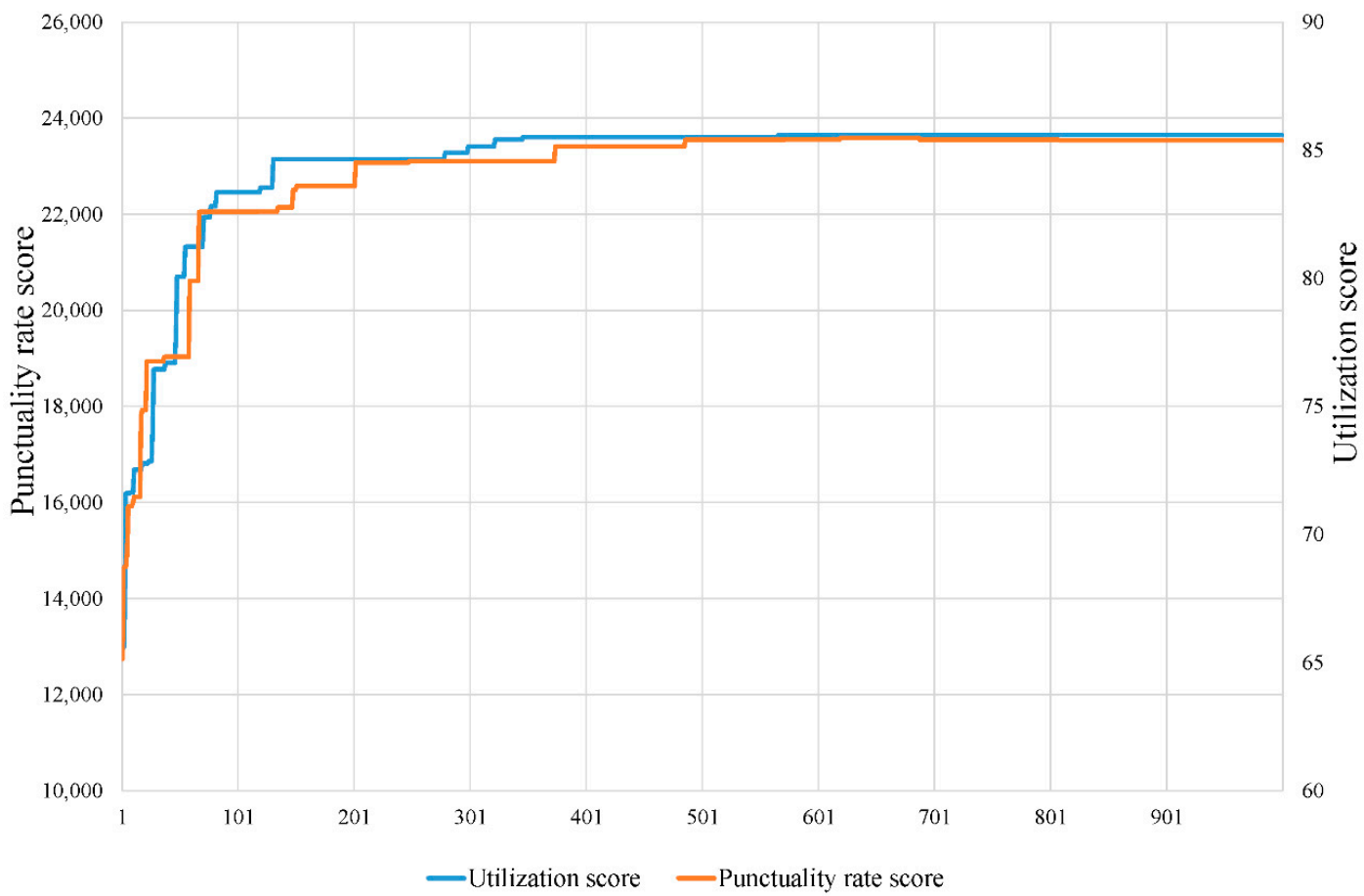

Figure 3. Two objective function score curves for each generation.

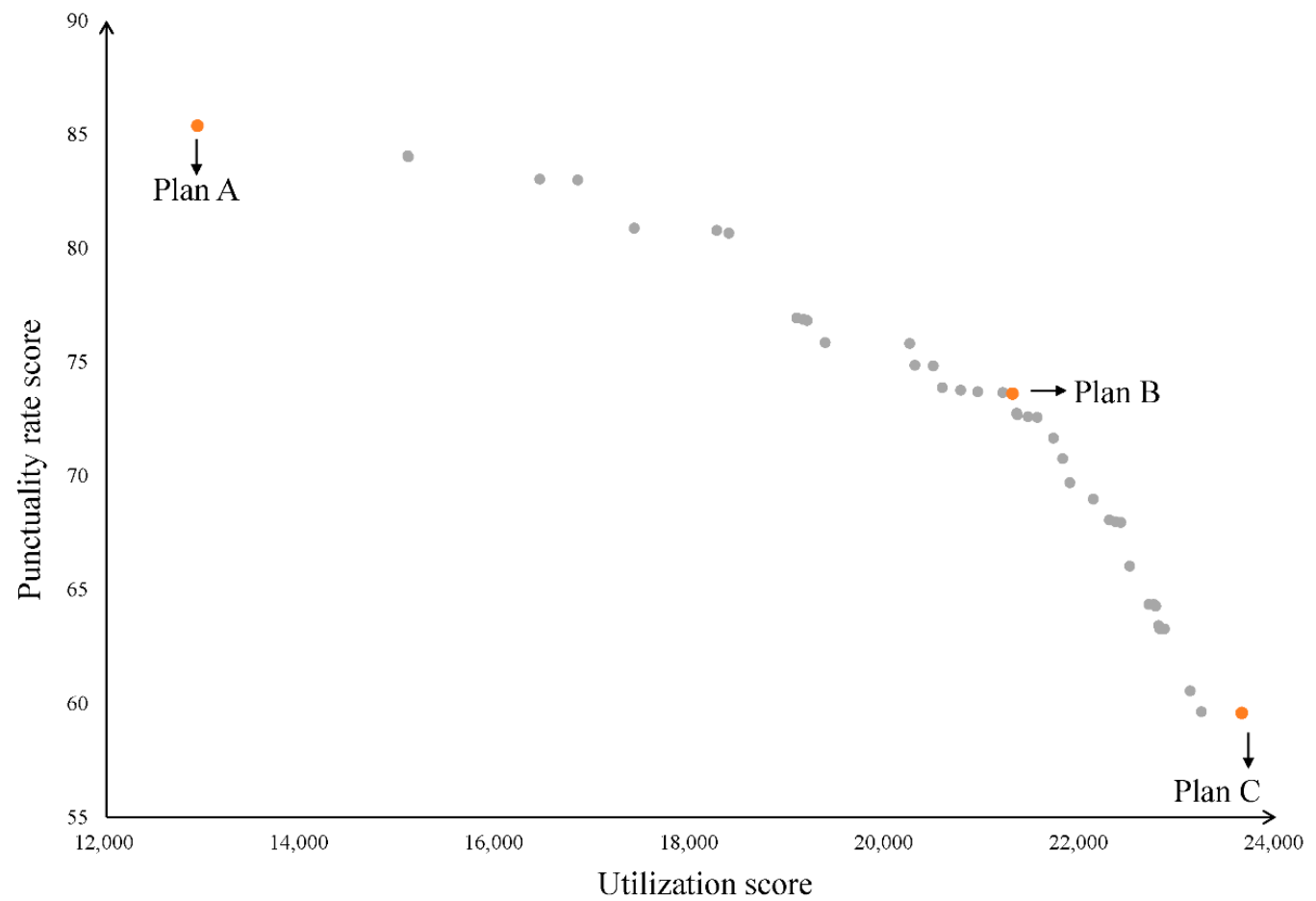

Figure 4. Final Pareto front solution set for bus lane planning. 
(a)

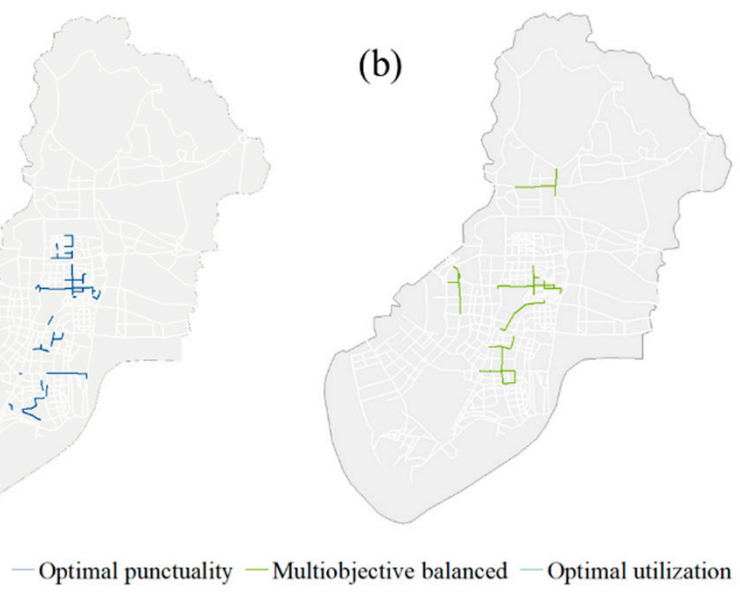

(c)

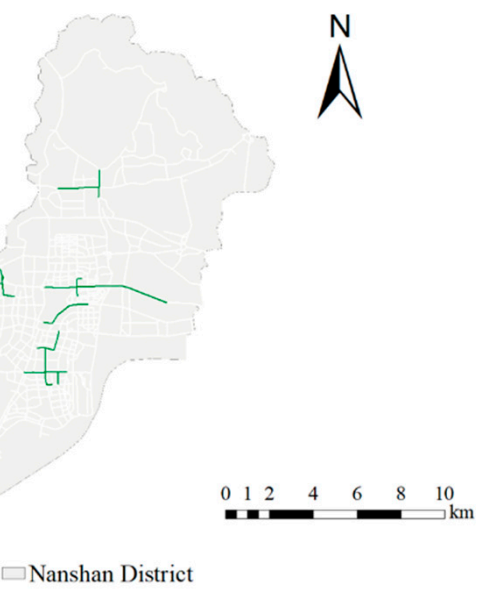

Figure 5. Bus lane planning results for Nanshan District achieved using the proposed method: (a) Plan A tends to have the best bus punctuality rate; (b) Plan B is a balance between punctuality and utilization; (c) Plan C tends to best optimize the utilization of bus lanes.

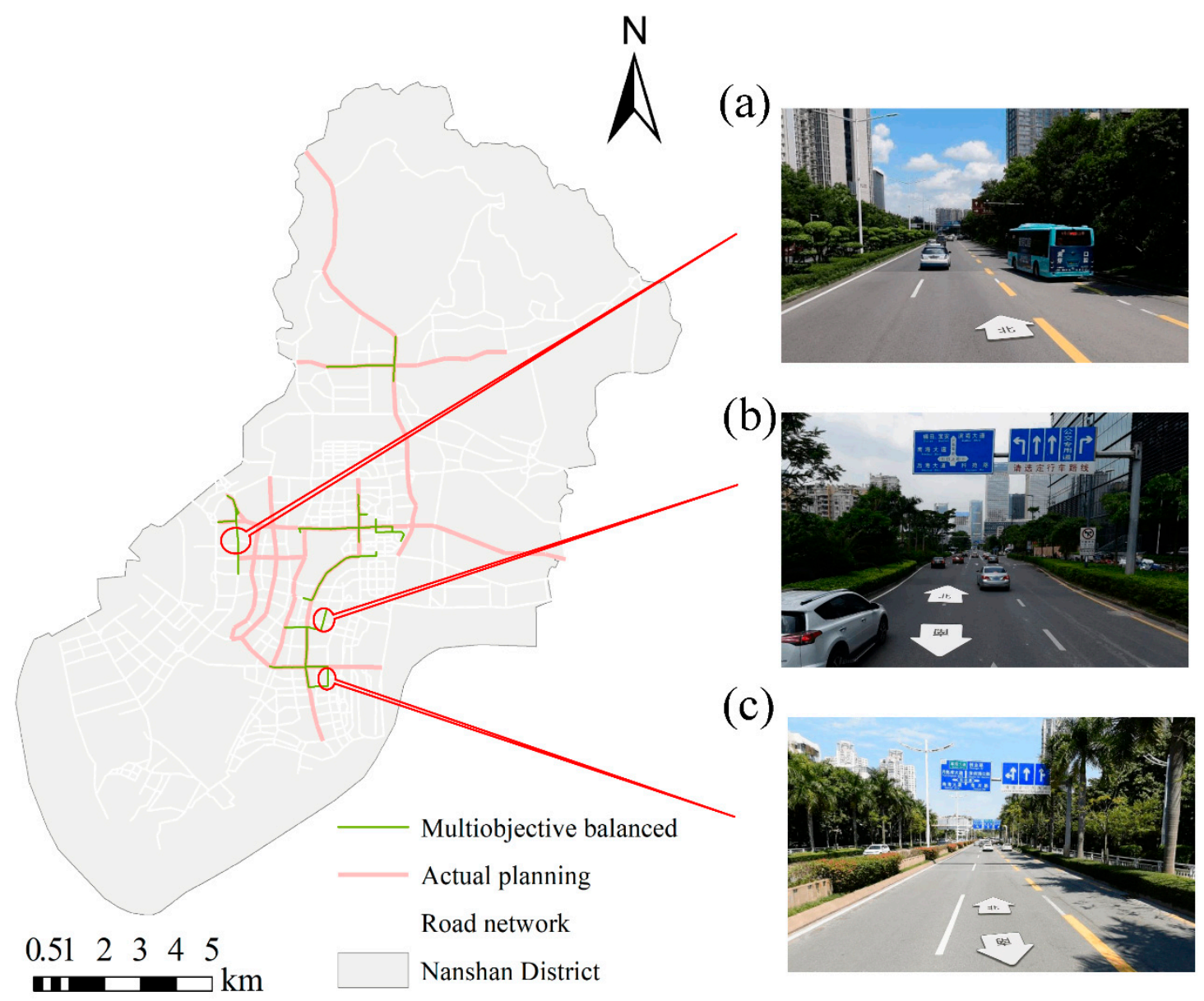

Figure 6. Comparison between the plans developed based on the proposed method and the actual planning scheme. (a-c) are the street views of three road segments that is in the red circle.

We also quantitatively compared the results of the two schemes. We calculated the length of the actual bus lane in Nanshan District, Shenzhen City, and used it as the construction budget constraint for bus lane planning. The statistics of the two objective functions for the actual planning scheme and the created planning scheme are shown 
in Figure 7. Both the utilization and punctuality scores suggest that the actual planning scheme is clearly worse than the scheme developed with the proposed method. The results also indicate that the proposed method can potentially be used to optimize existing bus lane planning.

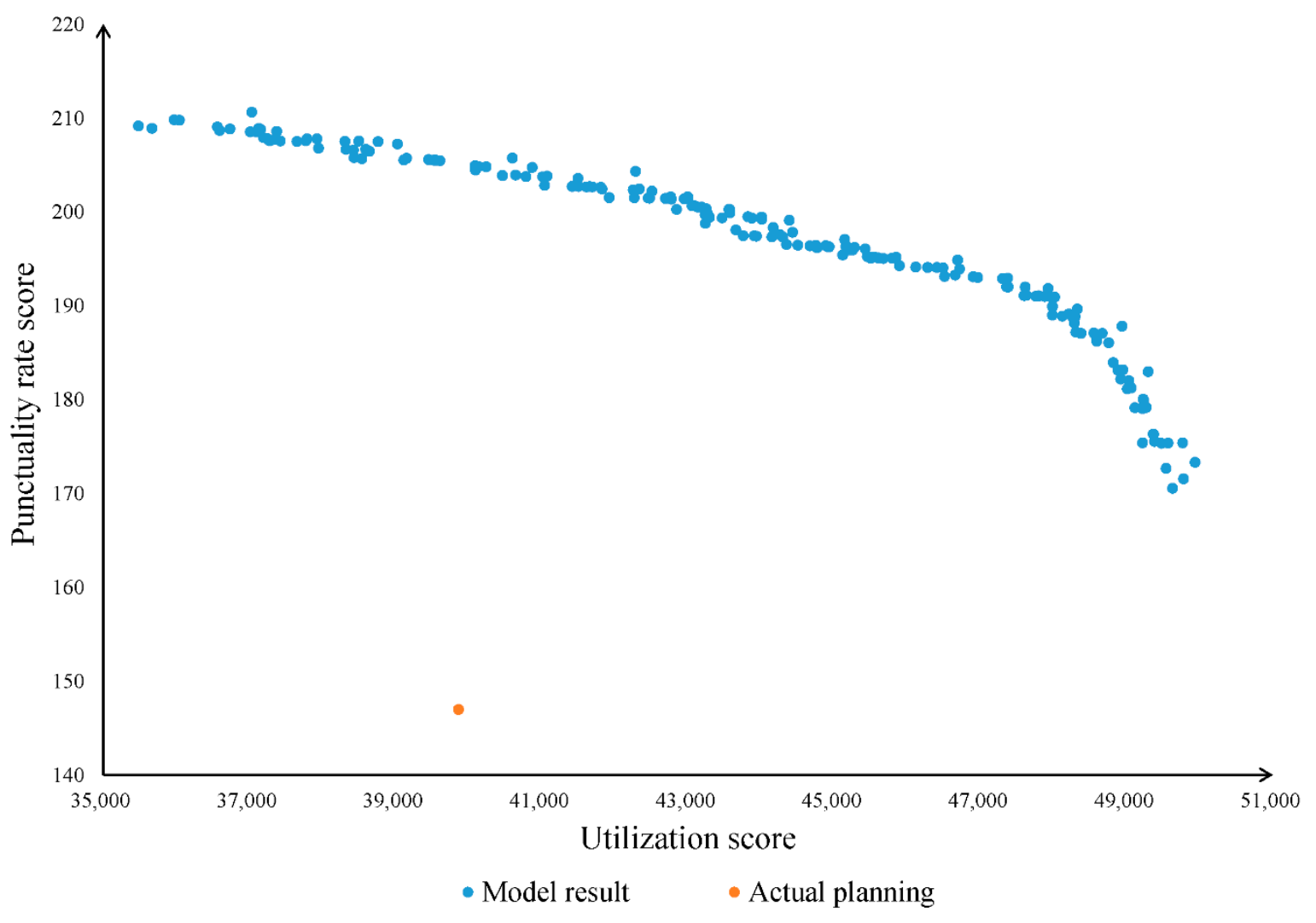

Figure 7. Comparison of the model results with actual planning scheme in Nanshan District, Shenzhen City.

\section{Discussion}

\subsection{Parameters}

Since a series of parameters, such as continuity constraints and construction budgets, are involved in the proposed method, we discuss their sensitivities and the corresponding effects on the results. We set the adjustment factor $\alpha=1.0$, the traffic flow constraint $f=90$ vehicles $/ \mathrm{h}$, the continuity constraint $k_{c}=6, k_{c n o n}=2$, and the construction budget $B=20 \mathrm{~km}$ to perform parameter analysis.

To examine the continuity constraint parameter, we set the continuity constraint to $k_{c}=0,3,6$, and 10 to plan the bus lanes and discuss the corresponding effect on the results. Figure 8 shows the Pareto front for different continuity constraint parameters. As the continuity constraint parameter increases, the punctuality rate function score shows an upward trend, and the utilization function score initially shows an upward trend. When the continuity constraint parameter $k_{c}$ reaches a certain value $(k=15)$, the scores are reduced. The utilization objective function involves the continuity of the bus trajectories. The existence of more continuous road segments increases the utilization score to a certain extent, but when the continuity constraint parameter reaches a certain level, the candidate road segments may be connected to continuous roads, which may lead to a lower score.

To examine the construction budget parameter, we set the construction budget to $B=$ 10,20 , and $40 \mathrm{~km}$ to plan the bus lanes and discuss the effect of the construction budget on the results. Figure 9 shows that as the construction budget increases, the Pareto front of the final result continues to advance in the direction of increasing the multiobjective function score, and the distribution of the solutions expands. Combined with the dotted 
line of the objective function extremum, the objective function scores show approximate linear growth with increasing construction budgets.

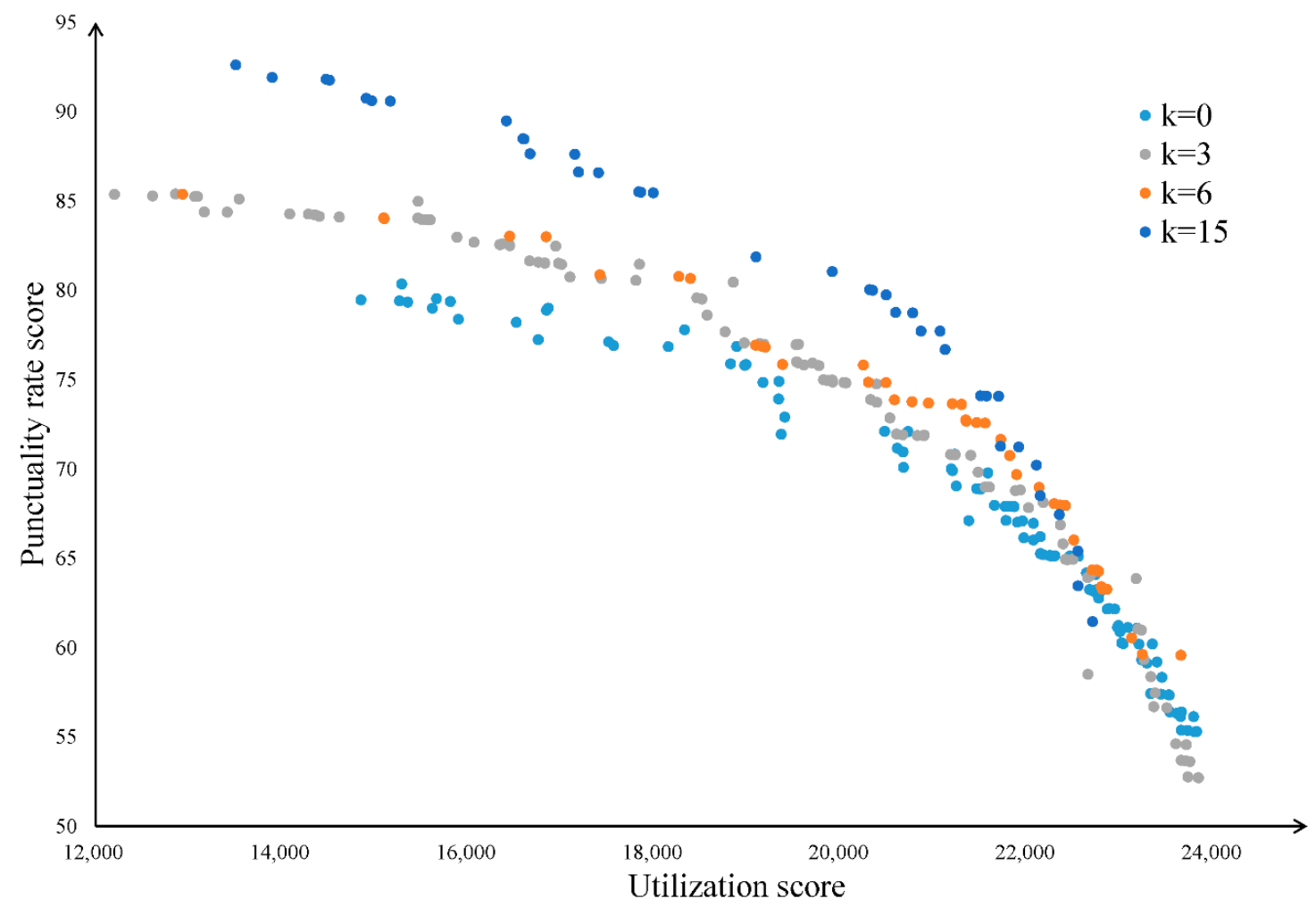

Figure 8. Planning results achieved with differing values of continuity constraint parameters $k$.

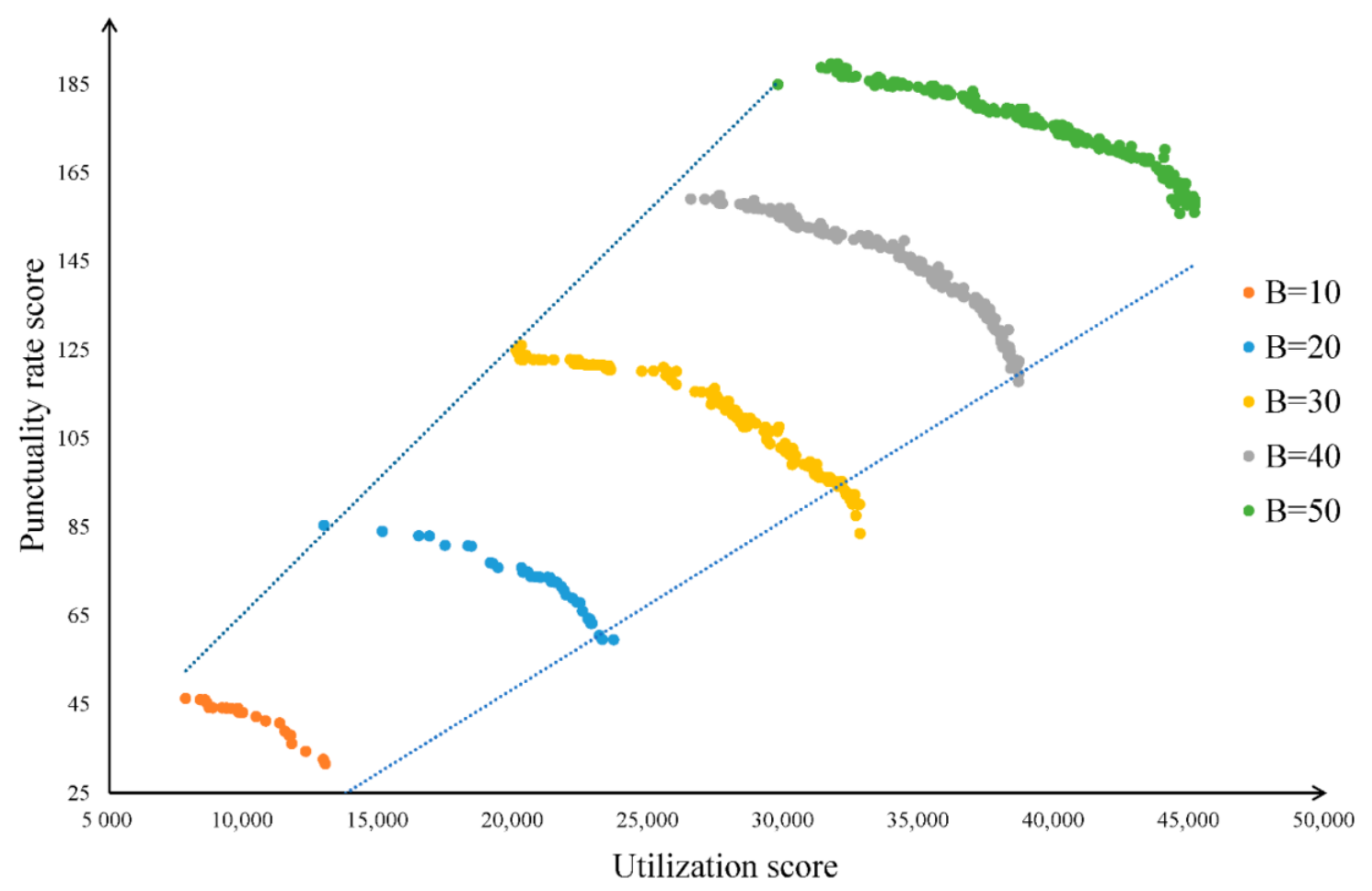

Figure 9. Planning results achieved with differing values of construction budgets $B(\mathrm{~km})$. 


\subsection{Quality}

This paper introduces a GPS-based method to plan bus lanes on an urban scale. The final quality is impacted by two main factors.

The first factor is the specification of traffic parameters. Except for road network data, the road/vehicle traffic parameters used by the model, such as the traffic flow, utilization, and coverage parameters, are specified based on the bus GPS trajectory data. Compared to that for historical experience and questionnaires, traffic parameter assignment based on GPS trajectories provides a more precise spatiotemporal resolution; furthermore, this approach can support high-precision and dynamic objective functions of road utilization and bus punctuality. Although this model does not use passenger flows directly, as is well known, the formulation of bus routes and bus schedules is based on passenger flows. In the model constraints and objective function, some parameters, such as the bus flow volume, bus frequency, and number of buses, which are positively correlated with passenger flows, are considered. Moreover, considering transit systems could be flexible and stretchy [15], although GPS trajectory can specify dynamic traffic information, it needs further research for this method to adapt to dynamic transit systems.

The second factor is the properties of the evolutionary algorithm. This paper treats bus lane planning as a multiobjective optimization problem and introduces NSGA2 to solve the problem. The algorithm requires $10 \mathrm{~min}$ to complete 1000 iterations and derive a series of candidate plans in the test area. It can therefore adapt to rapid changes in urban public transport and be conveniently deployed in other cities. In practice, there are many solution methods that can be further examined to obtain high-quality results. Unlike in single-objective optimization problems, the subgoals are contradictory in the multiobjective case and may produce a set of optimal solutions (Pareto-optimal solutions) [33]. Additionally, other multiobjective optimization methods have been used in existing bus lane planning models. For example, Hadas and Nahum [13] used the strengthened Pareto evolutionary algorithm 2 (SPEA2) to find the Pareto set for bus lane planning. The common multiobjective optimization methods that have different adaptabilities include particle swarm optimization (PSO) and artificial bee colony (ABC) algorithms [32]. Future work will include comparing these potential solution methods and the corresponding quality.

\section{Conclusions}

To fill the gap related to the lack of an adaptive method for bus lane planning, this paper proposes a new method that uses bus GPS trajectories to plan bus lanes. The bus lane planning problem is formulized as a multiobjective optimization problem. The constraints include the basic attributes of road segments, traffic flows, planned construction budget, and bus lane connectivity. For problem solving, considering the conflict between the multiple objectives and the efficiency of the algorithm, an evolutionary algorithm based on NSGA2 for the bus lane planning model is proposed. Finally, we illustrate the usability of the model in actual case studies and discuss the adaptability and effects of the input parameters of the model.

The main contribution of this article is the proposal of a GPS trajectory-driven bus lane planning method. Using GPS trajectories to obtain the traffic parameters is effective. Compared with the existing micromodels, the developed method can directly specify the traffic parameters for complex road networks at the city scale. Compared with the existing macromodels, the proposed model considers the traffic parameters for microscopic road segments, such as bus routes and the frequency and number of vehicles. Furthermore, in the objective function, a road utilization and bus punctuality function that is compatible with GPS trajectories is proposed, which provides the model with a better spatiotemporal resolution than traditional methods.

The article uses GPS trajectories in a data-driven approach to plan bus lanes. However, there are still several issues, such as not considering the impact of other vehicles on planned road segments and other objective functions. These factors need to be addressed in future research. 
Author Contributions: Conceptualization: Y.S. and M.W.; methodology: Y.S. and M.W.; software: Y.S. and H.L.; validation: Y.S., M.W., and H.L.; investigation: Y.S. and H.L.; resources: M.W.; data curation: M.W.; writing: Y.S. and M.W.; supervision: M.W.; project administration: M.W.; funding acquisition, M.W. All authors have read and agreed to the published version of the manuscript.

Funding: This research was funded by National Natural Science Foundation of China (41971417) and (41631175).

Institutional Review Board Statement: Not applicable.

Informed Consent Statement: Not applicable.

Data Availability Statement: The data and codes presented in this study are available from the corresponding author by request.

Conflicts of Interest: The authors declare no conflict of interest.

\section{References}

1. Gossling, S. Urban transport justice. J. Transp. Geogr. 2016, 54, 1-9. [CrossRef]

2. Deng, T.; Nelson, J.D. Recent Developments in Bus Rapid Transit: A Review of the Literature. Transp. Rev. 2011, 31, 69-96. [CrossRef]

3. Li, S.; Ju, Y. Evaluation of Bus-Exclusive Lanes. IEEE Trans. Intell. Transp. Syst. 2009, 10, 236-245. [CrossRef]

4. Chen, Q. An Optimization Model for the Selection of Bus-Only Lanes in a City. PLoS ONE 2015, 10, 0133951. [CrossRef]

5. Waterson, B.J.; Rajbhandari, B.; Hounsell, N.B. Simulating the impacts of strong bus priority measures. J. Transp. Eng. ASCE 2003, 129, 642-647. [CrossRef]

6. Ibarra-Rojas, O.J.; Delgado, F.; Giesen, R.; Munoz, J.C. Planning, operation, and control of bus transport systems: A literature review. Transp. Res. Part B Methodol. 2015, 77, 38-75. [CrossRef]

7. Seo, Y.; Park, J.; Jang, H.; Lee, Y. A Study on Setting-Up a Methodology and Criterion of Exclusive Bus Lane in Urban Area. In Proceedings of the Eastern Asia Society for Transportation Studies; J-Stage: Tokyo, Japan, 2005; pp. 339-351.

8. Mesbah, M.; Sarvi, M.; Currie, G. New Methodology for Optimizing Transit Priority at the Network Level. Transp. Res. Rec. J. Transp. Res. Board 2008. [CrossRef]

9. Si, B.; Ming, Z.; Yang, X.; Gao, Z. Bi-level Programming Model for Exclusive Bus Lanes Configuration in Multimodal Traffic Network. Transp. Res. Procedia 2017, 25, 652-663.

10. Khoo, H.L.; Teoh, L.E.; Meng, Q. A bi-objective optimization approach for exclusive bus lane selection and scheduling design. Eng. Optim. 2014, 46, 987-1007. [CrossRef]

11. Miandoabchi, E.; Farahani, R.Z.; Szeto, W.Y. Bi-objective bimodal urban road network design using hybrid metaheuristics. Cent. Eur. J. Oper. Res. 2012, 20, 583-621. [CrossRef]

12. Bagloee, S.A.; Ceder, A. Transit-network design methodology for actual-size road networks. Transp. Res. Part B Methodol. 2011, 45, 1787-1804. [CrossRef]

13. Hadas, Y.; Nahum, O.E. Urban bus network of priority lanes: A combined multi-objective, multi-criteria and group decisionmaking approach. Transp. Policy 2016, 52, 186-196. [CrossRef]

14. Chen, C.; Zhang, D.; Li, N.; Zhou, Z.H. B-Planner: Planning Bidirectional Night Bus Routes Using Large-Scale Taxi GPS Traces. IEEE Trans. Intell. Transp. Syst. 2014, 15, 1451-1465. [CrossRef]

15. Sayarshad, H.R.; Gao, H.O. Optimizing dynamic switching between fixed and flexible transit services with an idle-vehicle relocation strategy and reductions in emissions. Transp. Res. Part A Policy Pract. 2020, 135, 198-214. [CrossRef]

16. Xu, Z.; Cui, G.; Zhong, M.; Wang, X. Anomalous Urban Mobility Pattern Detection Based on GPS Trajectories and POI Data. ISPRS Int. J. Geo. Inf. 2019, 8. [CrossRef]

17. Han, S.S. Managing motorization in sustainable transport planning: The Singapore experience. J. Transp. Geogr. 2010, 18, 314-321. [CrossRef]

18. Hadas, Y.; Ceder, A. Optimal Connected Urban Bus Network of Priority Lanes. Transp. Res. Rec. J. Transp. Res. Board 2014, 2418, 49-57. [CrossRef]

19. Bao, J.; He, T.; Ruan, S.; Li, Y.; Zheng, Y. Planning Bike Lanes based on Sharing-Bikes' Trajectories. In Proceedings of the 23rd ACM SIGKDD International Conference on Knowledge Discovery and Data Mining, Halifax, NS, Canada, 13-17 August 2017; pp. 1377-1386.

20. Oldfield, R.H.; Bly, P.H. An analytic investigation of optimal bus size. Transp. Res. Part B Methodol. 1988, 22, 319-337. [CrossRef]

21. Bly, P.H.; Oldfield, R.H. An Analytic Assessment of Subsidies to Bus Services. Transp. Sci. 1986, 20, 200-212. [CrossRef]

22. Arampatzis, G.; Kiranoudis, C.T.; Scaloubacas, P.; Assimacopoulos, D. A GIS-based decision support system for planning urban transportation policies. Eur. J. Oper. Res. 2004, 152, 465-475. [CrossRef]

23. Truong, L.T.; Sarvi, M.; Currie, G. Exploring Multiplier Effects Generated by Bus Lane Combinations. Transp. Res. Rec. 2015, 2533, 68-77. [CrossRef] 
24. Xiaolin, L.; Jie, Y.; Nan, Z.; Haode, L. Optimization Model for Locating Continuous Exclusive Bus Lanes. J. Northeast. Univ. Nat. Sci. 2017, 38, 16-21.

25. Tse, L.Y.; Hung, W.T.; Sumalee, A. Bus lane safety implications: A case study in Hong Kong. Transp. A Transp. Sci. 2014, 10, 140-159. [CrossRef]

26. Yao, B.; Hu, P.; Lu, X.; Gao, J.; Zhang, M. Transit network design based on travel time reliability. Transp. Res. Part C 2014, 43, 233-248. [CrossRef]

27. Yu, B.; Kong, L.; Sun, Y.; Yao, B.; Gao, Z. A bi-level programming for bus lane network design. Transp. Res. Part C 2015, 55, 310-327. [CrossRef]

28. Barabino, B.; Di Francesco, M.; Mozzoni, S. Rethinking bus punctuality by integrating Automatic Vehicle Location data and passenger patterns. Transp. Res. Part A Policy Pract. 2015, 75, 84-95. [CrossRef]

29. Lin, N.-N.; Yang, X.-G.; Zhou, X.-M.; Xu, X. The Calculation of the Punctuality Rate between Bus Sites Based on AVL Data. In Proceedings of the 5th International Conference on Measuring Technology and Mechatronics Automation, Washington, DC, USA, 16-17 January 2013; pp. 1150-1152.

30. Wang, Z.; Lu, M.; Yuan, X.; Zhang, J.; Wetering, H.V.D. Visual Traffic Jam Analysis Based on Trajectory Data. IEEE Trans. Vis. Comput. Graph. 2013, 19, 2159-2168. [CrossRef]

31. Sila-Nowicka, K.; Vandrol, J.; Oshan, T.; Long, J.A.; Demsar, U.; Fotheringham, A.S. Analysis of human mobility patterns from GPS trajectories and contextual information. Int. J. Geogr. Inf. Sci. 2016, 30, 881-906. [CrossRef]

32. Kaim, A.; Cord, A.F.; Volk, M. A review of multi-criteria optimization techniques for agricultural land use allocation. Environ. Model. Softw. 2018, 105, 79-93. [CrossRef]

33. Deb, K. Multi-objective Optimization. In Search Methodologies: Introductory Tutorials in Optimization and Decision Support Techniques; Burke, E.K., Kendall, G., Eds.; Springer: Boston, MA, USA, 2014; pp. 403-449. [CrossRef]

34. Deb, K.; Pratap, A.; Agarwal, S.; Meyarivan, T. A fast and elitist multiobjective genetic algorithm: NSGA-II. IEEE Trans. Evol. Comput. 2002, 6, 182-197. [CrossRef]

35. Morais, H.; Sousa, T.; Castro, R.; Vale, Z. Multi-Objective Electric Vehicles Scheduling Using Elitist Non-Dominated Sorting Genetic Algorithm. Appl. Sci. 2020, 10, 7978. [CrossRef]

36. Hisao, I.; Noritaka, T.; Yusuke, N. Evolutionary many-objective optimization: A short review. In Proceedings of the IEEE Congress on Evolutionary Computation (IEEE World Congress on Computational Intelligence), Hong Kong, China, 1-6 June 2008; pp. 2419-2426.

37. Jain, H.; Deb, K. An Evolutionary Many-Objective Optimization Algorithm Using Reference-Point Based Nondominated Sorting Approach, Part II: Handling Constraints and Extending to an Adaptive Approach. IEEE Trans. Evol. Comput. 2014, 18, 602-622. [CrossRef]

38. Eichler, M.; Daganzo, C.F. Bus lanes with intermittent priority: Strategy formulae and an evaluation. Transp. Res. Part B Methodol. 2006, 40, 731-744. [CrossRef]

39. Li, Q.; Zhang, T.; Yu, Y. Using cloud computing to process intensive floating car data for urban traffic surveillance. Int. J. Geogr. Inf. Sci. 2011, 25, 1303-1322. [CrossRef]

40. Yuan, J.; Zheng, Y.; Zhang, C.; Xie, X.; Sun, G.Z. An Interactive-Voting Based Map Matching Algorithm. In Proceedings of the 11th International Conference on Mobile Data Management, Kansas City, MO, USA, 23-26 May 2010; pp. 517-520. 